\title{
A nose for cartilage repair
}

The results of a first-in-human study now published in The Lancet demonstrate that cartilaginous grafts engineered from autologous nasal chondrocytes can be used for the clinical repair of articular cartilage defects in the knee. "We have developed a promising approach to the treatment of articular cartilage injuries," reports corresponding author Ivan Martin, adding that further assessment in large cohorts and in randomized controlled trials with long-term assessment of clinical outcome is needed before this approach can be offered to patients as a standard treatment.

In this observational study, ten patients with post-traumatic full-thickness cartilage defects of the femoral condyle or trochlea were treated using grafts engineered from chondrocytes isolated from nasal cartilage biopsy specimens. Patients reported improvements in pain, knee function and quality of life, according to self-assessments performed before the surgical procedure and 24 months later."The main finding of the study, testing for the first time the use of nasal chondrocytes in patients' knees, is that the treatment is safe and feasible," says Martin. "Moreover, MRI data indicate that the tissue being formed at the patients' repair site improves in composition over time, getting more and more similar to healthy cartilage," he adds. "This result is by far not obvious, as it has not been demonstrated following alternative procedures ."

"Though employment of cartilage cells to engineer cartilage tissue is already in human use, the present study demonstrates that a relatively non-invasive source, nose cartilage, can be considered," comments Kyriacos Athanasiou, who was not involved in the study. He explains that cartilage grafts are usually engineered using chondrocytes from a cadaveric joint, or alternatively using cells taken from the patient's joint. "This study shows the use of non-articular cartilage cells taken directly from the patients themselves using a minimally invasive approach, to make grafts for clinical patients."

Earlier work by the same researchers had provided the rationale for using nasal chondrocytes for articular cartilage repair. "We previously demonstrated that nasal chondrocytes, harvested from the nasal septum, have a larger and more reproducible capacity to form new cartilage than articular chondrocytes, harvested from the knee joint," Martin recounts. "We further established that the cartilage tissue generated by nasal chondrocytes can respond to physical forces (mechanical loads) similar to articular cartilage and has the 'plasticity' to adapt to a joint environment, since it efficiently integrated with surrounding articular cartilage when implanted in goat joints."

"The results are exciting, though we need to keep in mind that this approach was used in young patients with small defects," says Athanasiou, commenting on the current study. "We also need to see long-term functional stability and integration of the grafts," he adds.

A phase II study based on the approach published in The Lancet is now underway, involving a total 108 patients at four centres in Switzerland, Germany, Croatia and Italy. Martin also suggests that further research should explore the possibility of using engineered nasal chondrocyte grafts for the treatment of degenerative pathologies, such as the early stages of osteoarthritis. "Extension of the treatment to these indications is currently being investigated in pre-clinical models in sheep, but the validation of the biological and pre-clinical basis to allow a first treatment in patients will still take a long time," he says.

Sarah Onuora

\section{ORIGINAL ARTICLE Mumme, M. et al.}

Nasal chondrocyte-based engineered autologous cartilage tissue for repair of articular cartilage defects: an observational first-in-human trial. Lancet 388, 1985-1994 (2016) 